\title{
Impact of Self Noise on Tracking Performance of Non-Data-Aided Digital Timing Recovery
}

\author{
Federica Scardoni, Maurizio Magarini, Member, IEEE, and Arnaldo Spalvieri.
}

\begin{abstract}
Non-data-aided feedforward timing recovery is preferred to non-data aided feedback timing recovery in digital receivers because it does not suffer of delay in the loop. However, while feedback timing recovery with optimized pre-detection filter is not affected by self noise, in this paper it is pointed out for the first time that, even if an optimized pre-detection filter is adopted, feedforward timing recovery is. The paper proposes an approximation to the power spectral density of self noise that leads to analytical performance evaluation, the accuracy of the approximation being confirmed by simulation results. Also, a comparative study of feedforward and feedback timing recovery is presented. The result of the comparison is that, for signal-to-noise ratio lower than $50 \mathrm{~dB}$ and realistic spectra of phase noise affecting the timing wave to be tracked, despite self noise, feedforward timing recovery outperforms feedback timing recovery.
\end{abstract}

Index Terms-Clock recovery, synchronization, timing jitter, self noise, phase noise.

\section{INTRODUCTION}

Non-data aided (NDA) digital timing recovery is a classical topic in communication theory that is receiving renewed interest in the framework of coherent optical communications, see e.g. [1]-[7]. The most popular digital NDA feedforward timing recovery scheme for Quadrature Amplitude Modulation (QAM) and Phase Shift Keying (PSK) modulation formats has been proposed by Oerder and Meyr in [8] where the timing detector makes use of the samples of the received signal at four times the symbol frequency. The four samples per symbol interval can be obtained either by sampling the time-continuous signal at four times the symbol frequency or by interpolating the samples taken at two times the symbol frequency as in [7], [9]-[12]. Timing detection with halfbaud spaced samples is performed also in the scheme due to Gardner [13], which is based on the Phase-Locked Loop (PLL) principle. Compared to the feedforward scheme, the feedback scheme suffers from delay in the feedback loop, which can be large in optical systems when digital signal processing is implemented in FPGA, compromising the performance of the PLL when it has large loop bandwidth [2], [7]. The need of large bandwidth arises when timing recovery has to track local oscillators affected by large phase noise, as the paper [4] has put in light in the context of feedforward timing recovery. More generally, the issue of delay in the loop is common to

Manuscript received Month DD, 2015; revised Month DD, 2015.

F. Scardoni is with Alcatel-Lucent, Vimercate, Italy (E-mail: federica_edvige.scardoni@alcatel-lucent.com).

$\bar{M}$. Magarini and A. Spalvieri are with the Dipartimento di Elettronica, Informazione e Bioingegneria, Politecnico di Milano, Italy (E-mail: \{maurizio.magarini,arnaldo.spalvieri\}@polimi.it). all the synchronization schemes based on the PLL principle, the impact of delay on system's performance being analyzed in [14] for PLL of first and second order.

In this paper we analyze the jitter performance of feedback and feedforward timing recovery in the presence of phase noise affecting the incoming timing wave and Additive White Gaussian Noise (AWGN). The main novelty of the paper is the evaluation by analytical means of the impact of self noise on the tracking performance, the analysis being validated by simulation results. The results of this paper show that, as conjectured in [4], self noise is responsible of the mismatch between the simulation results and the analysis of timing jitter when self noise is neglected. Specifically, it is shown in the paper that the power spectral density of self noise can be approximated to a slope of $+20 \mathrm{~dB} /$ decade, leading to a non-negligible contribution to the timing jitter when the postdetection filter (which will be called simply post-filter in the following) has large bandwidth, hence when the incoming timing wave to be tracked is affected by large phase noise. To achieve these results the paper improves over the existing literature as follows. The analysis of self noise spectrum generated by Gardner's detector, which is performed in [15] for Nyquist-type filters only, is extended here to a general form of pre-filter, thus allowing to take into account also optimized pre-filters as that of [16]. The cyclostationary spectrum of self noise generated by the square-law detector of the feedforward scheme is computed here for the first time. Based on the above results, the paper proposes to approximate self noise spectrum to a slope of $+20 \mathrm{~dB} /$ decade, also this approximation being proposed here for the first time. Finally, the paper derives optimal post-filters for the feedback and feedforward schemes in the presence of phase noise and their performance.

The outline of the paper is as follows. In Sec. II the system model is introduced. Section III reports the analysis of postdetection noise affecting the feedback scheme, while in Sec. IV the post-detection noise affecting the feedforward scheme is analyzed. Section V reports performance analysis and postfilter optimization for the feedback and feedforward schemes in the presence of phase noise affecting the incoming timing wave. Numerical results showing the close fit between analysis and simulations are presented in Section VI, while conclusions are drawn in Sec. VII.

\section{SySTEM MODEL}

Consider a continuous-time QAM or PSK baseband signal plus complex AWGN:

$$
r(t, \tau)=\sum_{k} a_{k} u(t-k T-\tau)+w(t),
$$


where $T$ is the symbol repetition interval which is assumed to be known, $\tau$ is a random and unknown parameter, $\left\{a_{k}\right\}$ is the sequence of i.i.d. complex random constellation points with zero mean and unit variance, $u(t)$ is the impulse response of the transmit filter, and $w(t)$ is complex AWGN with power spectral density $N_{0}$. We assume in what follows that the frequency response of the transmit filter $U(f)$ is the square root of a Nyquist filter bandlimited to $T^{-1}$. Although not strictly necessary, for concreteness we also assume that it has unit energy, so the impulse response of the Nyquist filter is 1 at time $t=0$, and we use the signal-to-noise ratio $\mathrm{SNR}=N_{0}^{-1}$ to characterize the AWGN channel. After the pre-detection filter (called simply pre-filter in what follows), which is also assumed to be bandlimited to $T^{-1}$, one has the signal

$$
y(t, \tau)=\sum_{k} a_{k} v(t-k T-\tau)+n(t),
$$

where $n(t)$ is the complex additive Gaussian noise at prefilter's output, and the impulse response $v(t)$ is the inverse Fourier transform of

$$
V(f)=U(f) P(f),
$$

where $P(f)$ is the frequency response of the pre-filter.

In the following two Sections we consider a fedback scheme based on the Gardner detector [13] and a feedforward scheme based on the Oerder and Meyr detector [8]. Both the detectors are known to be discrete-time approximations to the continuous-time square-law detector. Hence, the different behavior of the two schemes is induced by the feedback/feedforward implementation.

\section{FEEDBACK TIMING RECOVERY}

In the feedback scheme by Gardner [13], which is based on half-baud spaced sampling, the timing error detector is

$$
\Re\left\{y^{*}\left(k T-T / 2, \epsilon_{k}\right)\left(y\left(k T, \epsilon_{k}\right)-y\left((k-1) T, \epsilon_{k}\right)\right)\right\},
$$

where $\Re\{\cdot\}$ denotes the real part of $\cdot$, the superscript * denotes the complex conjugate, and

$$
\epsilon_{k}=\tau-\hat{\tau}_{k}
$$

being $\hat{\tau}_{k}$ the estimate of the timing instant produced at time $k$ by a PLL based on the phase detector (4).

At phaselock, that is when $E\left\{\epsilon_{k}\right\}=0$, the estimate of the timing instant is affected by a zero mean phase error

$$
\phi_{k}=\frac{2 \pi \epsilon_{k}}{T}
$$

whose autocorrelation in the $z$-domain can be written as

$$
R_{\phi}(z)=H(z) H^{*}\left(z^{-*}\right) \Psi(z),
$$

where we assume that the closed-loop transfer function $H(z)$ of the PLL has unit gain at $z=1$ and $z^{-*}$ is a shorthand for the complex conjugate of $z^{-1}$. The polynomial $\Psi(z)$ is the sum of the three terms "Noise $\times$ Noise," "Noise $\times$ Signal," "Signal $\times$ Signal" that come out when the sequence (2) is detected through (4):

$$
\Psi(z)=\Psi_{n \times n}(z)+\Psi_{n \times s}(z)+\Psi_{s \times s}(z) .
$$

In the following, we will assume that $\Psi_{s \times s}(1)=0$, an assumption that is met when the impulse response $p(t)$ of the pre-filter has certain symmetries that can be checked from the general form of $\Psi_{s \times s}(z)$ reported in Appendix A, for instance, when $p(t)$ is the inverse Fourier transform of a square-root Nyquist frequency response or when it is the impulse response of the optimized pre-filter of [16]. With this assumption, numerical evaluation of the spectrum for each of the three terms in (6) reveals that, while the slope of the power spectral density of the terms "Noise $\times$ Noise" and "Noise $\times$ Signal" at frequency zero is zero, the slope of the power spectral density of the term "Signal $\times$ Signal" at frequency zero is $+20 \mathrm{~dB} /$ decade. This motivates our proposed approximation

$$
\Psi(z) \approx \beta+\lambda(1-z)\left(1-z^{-1}\right)
$$

where

$$
\begin{gathered}
\beta=\beta_{n \times n}+\beta_{n \times s}, \\
\beta_{n \times n}=\Psi_{n \times n}(1)=\frac{2 \int_{0}^{T^{-1}}\left|P(f) P\left(T^{-1}-f\right)\right|^{2} d f}{T \cdot \mathrm{SNR}^{2} \cdot A^{2}}, \\
\beta_{n \times s}=\Psi_{n \times s}(1)=\frac{4 \int_{0}^{T^{-1}}\left|V(f) P\left(T^{-1}-f\right)\right|^{2} d f}{T^{2} \cdot \mathrm{SNR} \cdot A^{2}} \\
A=\left|\frac{4}{T} \int_{0}^{T^{-1}} e^{-j \pi f T} V(f) V^{*}\left(T^{-1}-f\right) d f\right| \\
\lambda=-\frac{1}{2} \sum_{m=-\infty}^{\infty} m^{2} \psi_{s \times s ; m},
\end{gathered}
$$

and the polynomial

$$
\Psi_{s \times s}(z)=\sum_{m=-\infty}^{\infty} \psi_{s \times s ; m} z^{-m}
$$

is given in appendix A. Equation (11) is obtained as follows. Our proposed approximation in frequency domain is

$$
\begin{aligned}
\Psi_{s \times s}\left(e^{j 2 \pi f T}\right) & \approx \lambda\left(1-e^{j 2 \pi f T}\right)\left(1-e^{j 2 \pi f T}\right) \\
& \approx \lambda(2 \pi f T)^{2}
\end{aligned}
$$

where the second approximation holds at low normalized frequency. The Fourier series expansion of the periodic even spectrum is given by

$$
\Psi_{s \times s}\left(e^{j 2 \pi f T}\right)=\sum_{m=-\infty}^{\infty} \psi_{s \times s ; m} \cos (2 \pi m f T),
$$

which, at low normalized frequency, is approximated to

$$
\Psi_{s \times s}\left(e^{j 2 \pi f T}\right) \approx \sum_{m=-\infty}^{\infty} \psi_{s \times s ; m}\left(1-\frac{(2 \pi m f T)^{2}}{2}\right) .
$$

Restricting our attention to pre-filters with symmetries such that $\Psi_{s \times s}(1)=0$, that is

$$
\sum_{m=-\infty}^{\infty} \psi_{s \times s ; m}=0
$$

and equating (12) to (13) one gets (11). 
For instance, let $U(f)$ be the square root of a raised-cosine Nyquist filter with roll-off $\alpha$ and assume that it has unit energy. When $P(f)$ is the filter matched to $U(f)$ and when it is the pre-filter of [16] one has

$$
\begin{gathered}
A=\frac{4 \sin (\pi \alpha / 2)}{\pi\left(4-\alpha^{2}\right)}, \\
\beta=\frac{\alpha \pi^{2}\left(4-\alpha^{2}\right)^{2}(1+\mathrm{SNR})}{16 \sin ^{2}\left(\frac{\pi \alpha}{2}\right) \cdot \mathrm{SNR}^{2}} .
\end{gathered}
$$

Also, one has $\lambda=0$ with the pre-filter of [16] and with the pre-filter of [17], while $\lambda$ can be computed numerically when the matched filter is used as a pre-filter.

\section{FEEDFORWARd TIMING RECOVERY}

In the feedforward scheme here considered, the timing detector produces the complex signal at symbol rate

$$
x_{k}(\tau)=\Re\left\{x_{k}(\tau)\right\}+j \Im\left\{x_{k}(\tau)\right\},
$$

where the real $(\Re)$ and imaginary $(\Im)$ parts are

$$
\begin{aligned}
& \Re\left\{x_{k}(\tau)\right\}=|y(k T, \tau)|^{2}-|y(k T-T / 2, \tau)|^{2}, \\
& \Im\left\{x_{k}(\tau)\right\}=|y(k T-T / 4, \tau)|^{2}-|y(k T-3 T / 4, \tau)|^{2},
\end{aligned}
$$

and we assume that the signal is sampled with frequency equal to four times the symbol frequency. The complex signal (16)(18) is passed through a feedforward post-filter, whose output at time $k$ is

$$
s_{k}(\tau)=A \cdot e^{j 2 \pi \tau / T}+\nu_{k}(\tau),
$$

where $\nu_{k}(\tau)$ is the complex cyclostationary noise and the amplitude of the desired complex exponential is

$$
A=\left|\frac{4}{T} \int_{0}^{T^{-1}} V(f) V^{*}\left(T^{-1}-f\right) d f\right| .
$$

The estimate of the timing instant at time $k$ is

$$
\hat{\tau}_{k}=\frac{T}{2 \pi} \arg \left\{s_{k}(\tau)\right\}=\tau+\frac{T}{2 \pi} \phi_{k}(\tau),
$$

where $\phi_{k}(\tau)$ is the cyclostationary phase error affecting the estimate.

When the power of $\nu_{k}(\tau)$ is much smaller than the power of the wanted complex exponential, the phase error that affects the estimate can be analyzed by writing the complex noise $\nu_{k}(\tau)$ of (19) in the form

$$
\nu_{k}(\tau)=\left(\nu_{r, k}(\tau)+j \nu_{t, k}(\tau)\right) e^{j 2 \pi \tau / T},
$$

where $\nu_{r, k}(\tau)$ and $\nu_{t, k}(\tau)$ are the noise components that are radial and tangential, respectively, to the desired complex exponential at time $k$. Equation (22) leads to

$$
\phi_{k}(\tau)=\arctan \left(\frac{\nu_{t, k}(\tau)}{A+\nu_{r, k}(\tau)}\right) \approx \frac{\nu_{t, k}(\tau)}{A} .
$$

Given the approximation (23), the cyclostationary $z$-spectrum of the phase error $\phi_{k}(\tau)$ can be written as

$$
R_{\phi}(z, \tau)=H(z) H^{*}\left(z^{-*}\right) \Psi(z, \tau),
$$

where we assume that the transfer function of the post-filter $H(z)$ has unit gain at $z=1$. Again, the polynomial $\Psi(z, \tau)$

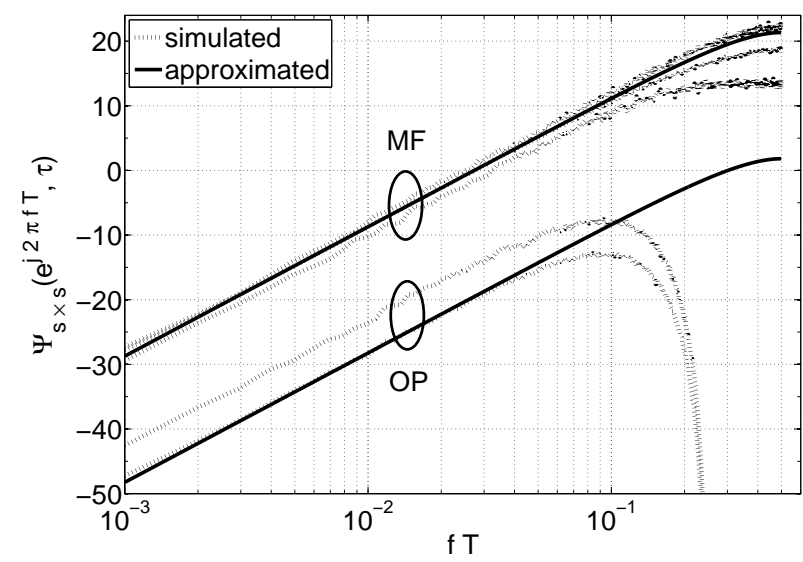

Fig. 1: Spectra of cyclostationary self noise with detector (17) (18), 256QAM, square-root raised cosine transmit filter with roll-off factor 0.25 , and two pre-filters: Matched Filter (MF) and Optimized Pre-filter of [16] (OP). Solid line: approximation with $\lambda$ from eqn. (28). Simulated spectra, MF, from the upper to the lower dotted line: worst $\tau$, average spectrum computed by (27), best $\tau$. Simulated spectra, OP, from the upper to the lower dotted line: worst $\tau$, average spectrum computed by (27). With the best $\tau$ the self noise spectrum with OP is zero [12].

can be written as the sum of the three terms "Noise $\times$ Noise," "Noise $\times$ Signal," "Signal $\times$ Signal" that come out when the sequence (2) is raised to the square:

$$
\Psi(z, \tau)=\Psi_{n \times n}(z, \tau)+\Psi_{n \times s}(z, \tau)+\Psi_{s \times s}(z, \tau) .
$$

The power spectral densities of the terms "Noise $\times$ Noise" and "Noise $\times$ Signal" at frequency zero are independent of $\tau$, and, with a further assumption of symmetry on the impulse response $v(t)$ [8], the power spectral density of the term "Signal $\times$ Signal" at frequency zero turns out to be equal to zero, but, as it happens in the feedback scheme, its slope is $+20 \mathrm{~dB} /$ decade, leading to the approximation

$$
\Psi(z, \tau) \approx \beta+\lambda(\tau)(1-z)\left(1-z^{-1}\right),
$$

where $\beta=\beta_{n \times n}+\beta_{n \times s}$ is given in equations (8) and (9), the term $\lambda(\tau)$ is obtained from the polynomial $\Psi_{s \times s}(z, \tau)$ given in Appendix B by a formula similar to (11). Again, assume that $U(f)$ is a unit energy square root raised-cosine Nyquist filter with roll-off $\alpha$. When $P(f)$ is the filter matched to $U(f)$ or the pre-filter of [16] one has

$$
\begin{gathered}
A=\frac{\alpha}{2}, \\
\beta=\frac{4(1+\mathrm{SNR})}{\mathrm{SNR}^{2} \alpha} .
\end{gathered}
$$

Phase noise always affects the local oscillators that generate the clocks that drive the data converters at the transmit and receive sides. An effect of phase noise is that, during time, $\tau$ randomly visits all the time instants in the period of the cyclostationary autocorrelation. This leads us to integrate the cyclostationary functions that depend on $\tau$ over the period $T$, leading to

$$
\Psi_{s \times s}(z)=\frac{1}{T} \int_{0}^{T} \Psi_{s \times s}(z, \tau) d \tau
$$


and

$$
\lambda=\frac{1}{T} \int_{0}^{T} \lambda(\tau) d \tau
$$

The excellent fit in the low frequency region between the actual $\Psi_{s \times s}\left(e^{j 2 \pi f T}\right)$ and its approximation can be appreciated from the spectra reported in Fig. 1 for two pre-filters. The worst and the best case of $\Psi_{s \times s}\left(e^{j 2 \pi f T}, \tau\right)$ for $\tau \in(0, T]$ are also reported in the same Figure.

The detector (17) (18) is a variant of the detector proposed by Oerder and Meyr [8]. Specifically, the detector considered here is the cascade of detector [8] and of an integrate-anddump filter over the symbol repetition interval. The integrateand-dump allows to get a symbol-spaced sequence at detector's output, as it happens with Gardner's detector (4). Moreover, equations (17) and (18) quickly lead to half-baud spaced detector of [10], [11], simply by replacing the right side of (18) by its approximation

$$
K \Re\left\{y^{*}(k T-T / 2, \tau)(y(k T, \tau)-y((k-1) T, \tau))\right\},
$$

where $K$ is the ratio between the right side of (20) and the right side of (10), [11].

\section{Performance with Phase Noise}

If the local oscillators that generate the clocks for data converters were free of phase noise, then the bandwidth of the post-filter could be vanishingly small, achieving virtually zero error between the actual timing instant and its estimate. Of course, this does not make sense, meaning in other words that sensible design of the post-detection filter must take phase noise into account. To make this possible, the random parameter $\tau$ appearing in (2) is hereafter replaced by the random sequence

$$
\tau_{k}=\frac{T}{2 \pi} \theta_{k}
$$

where $\left\{\theta_{k}\right\}$ is the phase noise sequence that affects the clocks that drive the data converters.

The objective in the design of the transfer function of the post-filter is to minimize the mean-square phase error

$$
E\left\{\phi_{k}^{2}\right\}=E\left\{\left(\theta_{k}-\hat{\theta}_{k}\right)^{2}\right\}
$$

where

$$
\hat{\theta}_{k}=\frac{2 \pi \hat{\tau}_{k}}{T} .
$$

Now the phase error $\phi_{k}$ is the sum of the post-detection noise passed through the post-filter and of the components of phase noise that are not recovered after the post-filter, hence the $z$ transform of its autocorrelation is

$$
\begin{aligned}
R_{\phi}(z) & =H(z) H^{*}\left(z^{-*}\right) \Psi(z) \\
& +(1-H(z))\left(1-H^{*}\left(z^{-*}\right)\right) \Upsilon(z)
\end{aligned}
$$

where $\Upsilon(z)$ is the $z$-spectrum of the phase noise sequence $\left\{\theta_{k}\right\}$ of (30).

The phase noise sequence $\left\{\theta_{k}\right\}$ to be tracked by the timing recovery mechanism is hereafter modelled as the discrete-time random walk

$$
\theta_{k+1}=\theta_{k}+\gamma n_{k}
$$

where $n_{k}$ is white Gaussian noise with zero mean and unit power, and $\gamma$ is the step size of the random walk. The $z$ transform of the autocorrelation of the random walk is

$$
\Upsilon(z)=\frac{\gamma^{2}}{\left(1-z^{-1}\right)(1-z)}
$$

For instance, a passively mode locked laser oscillator at frequency $T^{-1}=40 \mathrm{GHz}$ is characterized by $\gamma^{2}=4 \cdot 10^{-6}$, leading to a power spectral density of phase noise at $100 \mathrm{kHz}$ from the peak equal to $-64 \mathrm{dBcarrier} / \mathrm{Hz}$ [18], while an active mode-locked laser oscillator, that has better quality than the passive one, has $\gamma^{2}=10^{-8}$, that is $-90 \mathrm{dBcarrier} / \mathrm{Hz}$ at 100 $\mathrm{kHz}$ from the peak of the spectral line [19]. An even better phase noise spectrum can be obtained by a system based on a VCO with natural frequency around $10 \mathrm{GHz}$ locked to a reference crystal oscillator [20].

\section{A. Optimization of the Loop Filter in the Feedback Scheme}

In this subsection, optimization of the 1-causal loop filter is considered. This is the case where the delay in the loop is the minimum one, that is, one symbol repetition interval. In practical cases the loop delay can be much larger. Large loop delay would limit the loop bandwidth, thus compromising performance as studied in [5]. In other words we are analyzing the best performance achievable with the feedback scheme.

Following the method by Bode and Shannon, first of all we compute the whitening filter $W(z)$ of the input sequence as the causal and minimum phase transfer function resulting from the spectral factorization

$$
W(z) W^{*}\left(z^{-*}\right)=\frac{1}{\Psi(z)+\Upsilon(z)} .
$$

Using (7) and (34) for the $z$-spectra of noise and signal and performing the spectral factorization one finds

$$
\frac{1}{\Upsilon(z)+\Psi(z)}=\frac{\xi^{2}\left(1-z^{-1}\right)(1-z)}{\prod_{i=1}^{2}\left(1-z_{i} z^{-1}\right)\left(1-z_{i}^{*} z\right)},
$$

where

$$
\begin{gathered}
\xi^{2}=\frac{z_{1} z_{2}}{\lambda} \\
z_{1}, z_{1}^{-*}=-\frac{1}{2 \sqrt{2}}\left(-\frac{\beta^{3}}{\lambda^{2} P}+\frac{\beta^{2}}{\lambda^{2}}+\frac{4 \beta\left(\gamma^{2}-\beta\right)}{\lambda P}+\right. \\
\left.+\frac{16 \gamma^{2}}{P}+\frac{4 \beta-2 \gamma^{2}}{\lambda}\right)^{1 / 2}-\frac{P+Q}{4 \lambda} \\
z_{2}, z_{2}^{-*}=-\frac{1}{2}\left(\frac{Q^{2}}{2 \lambda^{2}}+\frac{1}{2 P}\left(-\frac{Q^{3}}{\lambda^{2}}+\frac{4 Q R}{\lambda}-8 Q\right)+\right. \\
\left.-\frac{R}{2}-2\right)^{1 / 2}+\frac{P-Q}{4 \lambda},
\end{gathered}
$$

with $P=\sqrt{\beta^{2}-4 \lambda \gamma^{2}}, Q=-4 \lambda-\beta$ and $R=6 \lambda+\gamma^{2}+2 \beta$. Selecting for $z_{1}$ and $z_{2}$ the two roots lying inside the unit circle one gets

$$
\begin{aligned}
& W(z)=\frac{\xi\left(1-z^{-1}\right)}{\left(1-z_{1} z^{-1}\right)\left(1-z_{2} z^{-1}\right)}, \\
& W^{*}\left(z^{-*}\right)=\frac{\xi(1-z)}{\left(1-z_{1}^{*} z\right)\left(1-z_{2}^{*} z\right)} .
\end{aligned}
$$


Finally, for the optimal transfer function one has

$$
H(z)=W(z)\left[\Upsilon(z) W^{*}\left(z^{-*}\right)\right]^{+},
$$

where the notation $[P(z)]^{+}$indicates the 1 -causal part of $P(z)$, that is

$$
[P(z)]^{+}=\sum_{k=1}^{\infty} p_{k} z^{-k}
$$

Substituting (34) and (38) in the bracketed term appearing in the right side of (39) one finds

$$
\begin{aligned}
{\left[\Upsilon(z) W^{*}\left(z^{-*}\right)\right]^{+} } & =\left[\frac{\xi \gamma^{2}}{\left(1-z^{-1}\right)\left(1-z_{1}^{*} z\right)\left(1-z_{2}^{*} z\right)}\right]^{+} \\
& =\frac{\xi \gamma^{2} \zeta^{2} z^{-1}}{\left(1-z^{-1}\right)}
\end{aligned}
$$

where

$$
\zeta^{2}=\frac{1}{\left(1-z_{1}\right)\left(1-z_{2}\right)}=\sqrt{\frac{1}{\gamma^{2} \xi^{2}}} .
$$

By replacing (37), (40), and (41), in (39) one gets

$$
H(z)=\frac{\xi \gamma z^{-1}}{\left(1-z_{1} z^{-1}\right)\left(1-z_{2} z^{-1}\right)} .
$$

The above transfer function can be implement by a PLL whose open loop transfer function, after straightforward algebra, is

$$
G(z)=\frac{H(z)}{1-H(z)}=\frac{\xi \gamma z^{-1}}{z_{1} z_{2} z_{p}^{-1}\left(1-z^{-1}\right)\left(1-z_{p} z^{-1}\right)},
$$

where

$$
z_{p}=\frac{2 z_{1} z_{2}}{z_{1}+z_{2}+\gamma \xi+\sqrt{\left(z_{1}+z_{2}+\gamma \xi\right)^{2}-4 z_{1} z_{2}}} .
$$

\section{B. Optimization of the Post-Filter in the Feedforward Scheme}

By standard arguments one finds that the transfer function of the post-filter that minimizes the mean-square error in the feedforward scheme is

$$
H(z)=\frac{\Upsilon(z)}{\Upsilon(z)+\Psi(z)},
$$

and that the $z$-spectrum of the estimation error is

$$
R_{\phi}(z)=\frac{\Upsilon(z) \Psi(z)}{\Upsilon(z)+\Psi(z)} .
$$

Substituting (26) and (27) for the spectrum of detector's noise and (34) for the spectrum of phase noise affecting the incoming timing wave into (45) and (46) one finds

$$
\begin{aligned}
H(z) & =\frac{\gamma^{2}}{\gamma^{2}-\left(z-2+z^{-1}\right)\left(\beta-\lambda\left(z-2+z^{-1}\right)\right)}, \\
R_{\phi}(z) & =\frac{\gamma^{2}\left(\beta-\lambda\left(z-2+z^{-1}\right)\right)}{\gamma^{2}-\left(z-2+z^{-1}\right)\left(\beta-\lambda\left(z-2+z^{-1}\right)\right)} .
\end{aligned}
$$

Unfortunately, implementing the post-filter with transfer function (47) is difficult, because its impulse response is a twosided decaying function with two-sided infinite duration. In the Section devoted to the numerical results, we will consider as a post-filter the classical moving average filter of [8] and the cascade of two moving average filters proposed in [4].

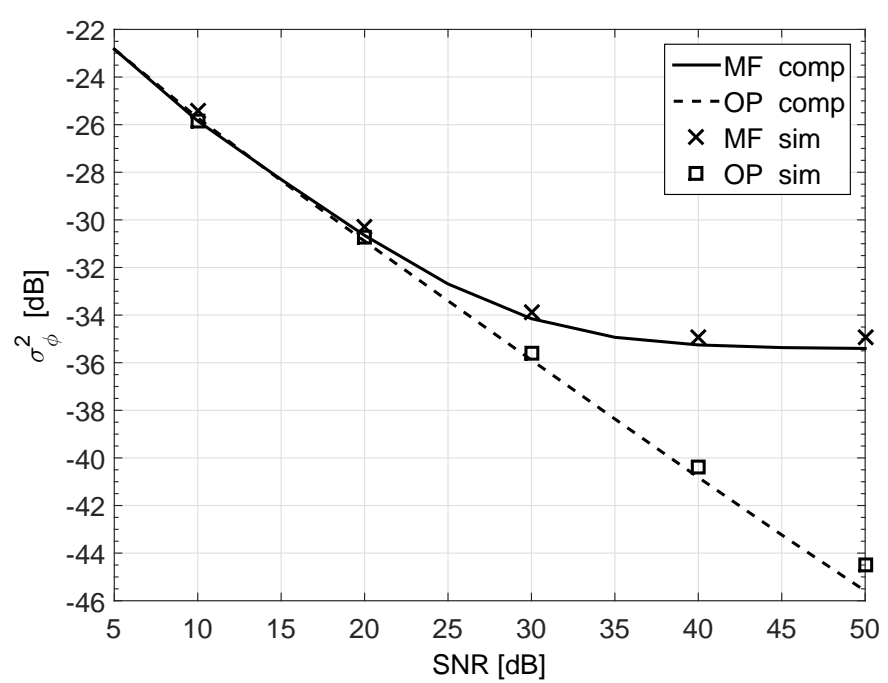

Fig. 2: Phase error variance versus SNR for the feedback scheme with the optimal loop filter (43). Solid line: computed variance with matched receive filter. Dotted line: computed variance with the optimized prefilter of [16]. Crosses: simulation results with matched filter. Squares simulation results with optimized pre-filter.

\section{NUMERICAL RESULTS}

The system considered in this Section is based on 256QAM with square-root raised-cosine transmit filter with rolloff factor equal to 0.25 . The phase noise parameter $\gamma^{2}$ is set to $4 \cdot 10^{-6}$, a value that, from the literature we are aware of, seems to be a worst case for real-world systems. Two types of pre-filters are considered: the square-root raised-cosine prefilter matched to the transmit filter and the optimized pre-filter of [16]. The mean-square phase error

$$
\sigma_{\phi}^{2}=T \int_{0}^{T^{-1}} R_{\phi}\left(e^{j 2 \pi f T}\right) d f
$$

is computed numerically using and adopted as a performance measure. The use of the mean-square phase error as a performance measure is motivated as follows. The SNR penalty due to the inter-symbol interference caused by timing jitter is

$$
10 \cdot \log _{10}(1+\mathrm{SNR} \cdot \mathrm{eMSE}) \mathrm{dB},
$$

where the excess MSE (eMSE) can be computed from the mean-square phase error as (see [4])

$$
\mathrm{eMSE}=\frac{\sigma_{\phi}^{2}}{4 \pi^{2}} \sum_{k=-\infty}^{\infty}(\dot{g}(k T))^{2}+\mathrm{HOT},
$$

where $\dot{g}(t)$ is the first time derivative of the Nyquist impulse response $g(t)$, and HOT stands for Higher Order Terms. With raised-cosine Nyquist filter with roll-off $\alpha$ one has

$$
\sum_{k=-\infty}^{\infty}(\dot{g}(k T))^{2}=\frac{6 \alpha^{2}\left(\pi^{2}-8\right)-3 \pi^{2} \alpha+2 \pi^{2}}{6} .
$$

For instance, with $\alpha=0.25, \mathrm{SNR}=25 \mathrm{~dB}$, and $\sigma_{\phi}^{2}=-38 \mathrm{~dB}$, neglecting the higher order terms one has an SNR penalty of $0.04 \mathrm{~dB}$.

The results obtained with the feedback scheme and optimal post-filter, using in (49) eqns. (32) and (42), are reported in Fig. 2. The floor that appears when one renounces to 


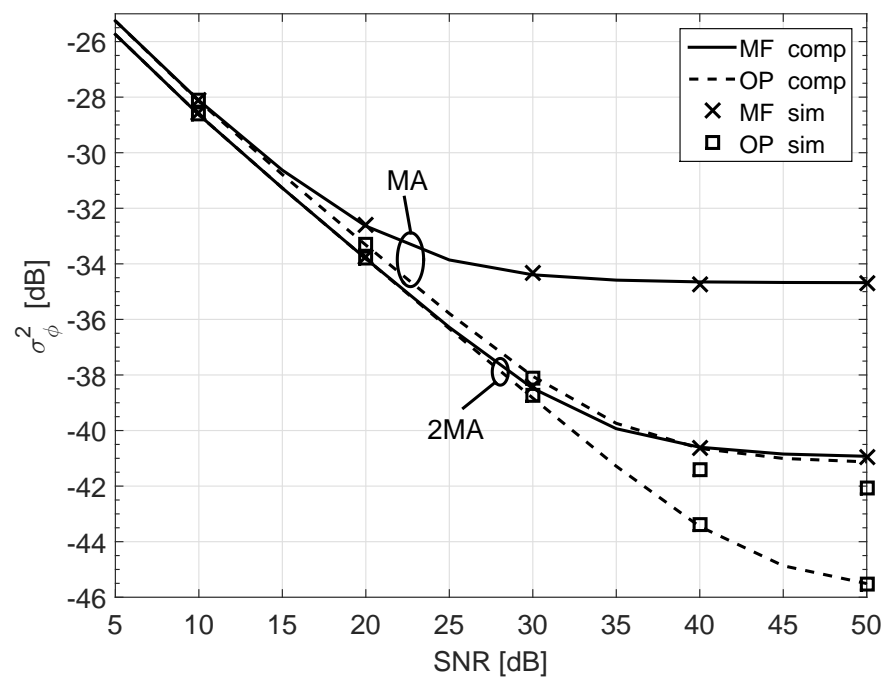

Fig. 3: Phase error variance versus SNR for the feedforward scheme. Moving Average filter (MA) and cascade of two Moving Averages filters (2MA) are used as post-filters. Solid line: computed variance with matched receive filter. Dotted line: computed variance with the optimized pre-filter of [16]. Crosses: simulation results with matched filter. Squares simulation results with optimized pre-filter.

the optimized pre-filter is due to self noise. The fit between simulations and analysis is excellent. With optimized pre-filter, there is only a moderate mismatch at SNR $=50 \mathrm{~dB}$. The mismatch is due to the wandering of the phase error $\phi_{k}$ around zero. Actually, when $\phi_{k}$ is not zero, the optimal pre-filter does no more guarantee ideal suppression of self noise.

The results obtained with the feedforward scheme are reported in Fig. 3, where, for each one of the two pre-filters, the cascade of two moving average filters proposed in [4] and the classical moving average filter proposed in [8] are used as post-filters. The duration of the impulse response of the postfilter is optimized to minimize the mean-square phase error. Fixed the post-filter, as expected from [12] the optimized prefilter outperforms the matched filter. The results also show that the cascade of two moving average filters outperforms the classical moving average filter, especially at high SNR. Actually, at high SNR the bandwidth of the post-filter becomes large, and the high-frequency portion of the spectrum of self noise is not well rejected by the tails of the frequency response of the moving average filter, while the cascade of two moving average filters is much more effective in rejecting the undesired high frequency portion of the spectrum of self noise. Noticeably, the performance with the cascade of two moving average filters is virtually insensitive to the pre-filter in the low-to-intermediate SNR region, say up to SNR equal to about $30 \mathrm{~dB}$, that is a reasonable SNR value for the threshold of a coded 256-QAM. At SNR $=30 \mathrm{~dB}$ the mean-square phase error with post-filter based on 2 moving average filters is about $-38 \mathrm{~dB}$, leading to, as shown before, an SNR penalty of only $0.04 \mathrm{~dB}$. With smaller $\gamma^{2}$, the divergence between the performance of matched pre-filter and optimized prefilter occurs at SNRs even higher than $30 \mathrm{~dB}$, leading to the conclusion that, with modulation formats as dense as 256QAM or less, there is no need of using an optimized prefilter. From Fig. 3 it can also be seen that simulation results confirm the analysis based on (27) and on the approximation (26). The small disagreement between analysis and simulation that is observed at high SNR for the case of optimized prefilter and moving average post-filter can be explained by observing that the approximation of the spectrum of self noise used in the analysis over-estimates the actual spectrum in the high-frequency region, as it can be seen from Fig. 1. When filtered through the high tails of the moving average filter, this mismatch induces the disagreement of about $1 \mathrm{~dB}$ between the square and the dotted line that can be seen at $\mathrm{SNR}=50 \mathrm{~dB}$ in Fig. 3. We have checked that the performance of the cascade of two moving average filters is within 0.07 to 0.2 decibels from the performance of the optimal post-filter (47). Also, we have simulated the performance of the detector of [11], which turns out to be virtually indistinguishable from the performance of the detector (17), (18).

\section{CONCLUSION}

The power spectral density of self noise in non-data-aided feedback and feedforward timing recovery has been studied in the paper. The presented results show that, while in the feedback scheme an optimized pre-filter can suppress self noise, in feedforward timing recovery self noise cannot be completely suppressed. Since feedforward timing recovery is preferred to feedback timing recovery in digital receivers, these results push to deepen the investigation.

What we have found about the feedforward scheme is that, even if an optimized pre-filter is adopted, the power spectral density of self noise can be suppressed only at frequency zero, then, at higher frequency, it can be approximated to a slope of $+20 \mathrm{~dB} /$ decade. Therefore, when the bandwidth of the post-filter is large, large bandwidth being a need when the timing wave to be tracked is affected by large phase noise, the impact of self noise on the performance of feedforward timing recovery cannot be neglected.

The analytical results presented in the paper, confirmed by computer simulations, show that, with a bad phase noise spectrum $(-64 \mathrm{dBcarrier} / \mathrm{Hz} @ 100 \mathrm{kHz})$, the impact of self noise can be non-negligible when the square-root Nyquist prefilter with a simple moving average post-filter as in [8] is used. Conversely, with the post-filter based on the cascade of two moving average filters proposed in [4], even if the conventional square-root Nyquist filter is used as a pre-filter, the impact of self noise is negligible for constellations as dense as 256 QAM or less. Moreover, our results also show that feedforward timing recovery with square-root Nyquist prefilter outperforms feedback timing recovery with optimized pre-filter for SNR lower than $40 \mathrm{~dB}$, while, if optimized pre-filter is used also in the feedforward scheme, then it outperforms the feedback scheme up to $\mathrm{SNR}=50 \mathrm{~dB}$, that is, in all the cases of practical interest that can be foreseen today. By computing the excess mean-square error induced by the feedforward scheme with better phase noise spectra, such as those of [19] and [20], one finds that clock recovery does not impact system performance. This also holds for the feedback scheme provided that the loop delay is small enough to allow for the desired loop bandwidth, see [5]. 


\section{APPENDIX A}

Let

$$
\Psi_{s \times s}(z)=\sum_{k=-\infty}^{\infty} \psi_{s \times s ; k} z^{-k} .
$$

The coefficients of the above polynomial can be written as

$$
\psi_{s \times s ; m}=\psi_{1 ; m}-\psi_{2 ; m}-\psi_{3 ; m}
$$

where

$$
\begin{gathered}
\psi_{1, m}=\delta_{m}\left(2 B v^{2}\left(\frac{T}{2}\right)+2 C \sum_{l=-\infty}^{\infty} v^{2}\left(l T-\frac{T}{2}\right)\right) \\
\psi_{2, m}=\left(\delta_{m-1}+\delta_{m+1}\right) \cdot \\
\left(B v^{2}\left(\frac{T}{2}\right)+C \sum_{l=-\infty}^{\infty} v\left(l T-\frac{T}{2}\right) v\left(l T+\frac{T}{2}\right)\right) \\
\psi_{3, m}=\sum_{l=-\infty}^{\infty} \delta_{m-l} \cdot C \cdot\left(v\left(m T+\frac{T}{2}\right)-v\left(m T-\frac{T}{2}\right)\right)^{2},
\end{gathered}
$$

where

$$
\begin{gathered}
\delta_{m}= \begin{cases}1, & m=0, \\
0, & m \neq 0,\end{cases} \\
B=\frac{A_{4}-3}{2 A^{2}}, \quad C=\frac{1}{2 A^{2}},
\end{gathered}
$$

with

$$
A_{4}=2\left(E\left\{\left(\Re\left\{a_{k}\right\}\right)^{4}\right\}+E\left\{\left(\Im\left\{a_{k}\right\}\right)^{4}\right\}\right) .
$$

Using the above coefficients in (11) one has

$$
\begin{aligned}
\lambda= & B v^{2}\left(\frac{T}{2}\right)+C \sum_{l=-\infty}^{\infty} v\left(l T-\frac{T}{2}\right) v\left(l T+\frac{T}{2}\right) \\
& +C \sum_{l=1}^{\infty} l^{2}\left(v\left(l T+\frac{T}{2}\right)-v\left(l T-\frac{T}{2}\right)\right)^{2} .
\end{aligned}
$$

\section{APPENDIX B}

Although being quite straightforward, exact computation of $\Psi_{s \times s}(\tau, z)$ is lengthy and tedious, therefore we report only the final results (for details see [21].) Let

$$
\Psi_{s \times s}(z, \tau)=\sum_{k=-\infty}^{\infty} \psi_{s \times s ; k}(\tau) z^{-k} .
$$

The coefficients of the above polynomial are as follows.

$$
\begin{array}{r}
\psi_{s \times s ; m}(\tau)=\frac{1}{2 A^{2}}\left(R_{1}(\tau, m T) \sin ^{2}\left(\frac{2 \pi \tau}{T}\right)+\right. \\
+R_{1}(\tau-T / 4, m T) \cos ^{2}\left(\frac{2 \pi \tau}{T}\right)+ \\
\left.-\frac{1}{2}\left(R_{2}(\tau, m T)+R_{2}(\tau,-m T)\right) \sin \left(\frac{4 \pi \tau}{T}\right)\right),
\end{array}
$$

where

$$
\begin{aligned}
R_{1}(\tau, m T)= & \left(A_{4}-3\right) R_{1, a}(\tau, m T)+ \\
& +2 R_{1, b}(\tau, m T)+2 R_{1, c}(\tau, m T), \\
R_{2}(\tau, m T)= & \left(A_{4}-3\right) R_{2, a}(\tau, m T)+ \\
& +2 R_{2, b}(\tau, m T)+2 R_{2, c}(\tau, m T) .
\end{aligned}
$$

The above $R_{1,\{a, b, c\}}(\tau, m T)$ and $R_{2,\{a, b, c\}}(\tau, m T)$ are

$$
\begin{aligned}
& R_{1, a}(\tau, m T)=\sum_{i} q_{m}^{2}(i T+\tau)+\sum_{i} q_{m}^{2}(i T+\tau-T / 2) \\
& -\sum_{i} q_{m-1 / 2}^{2}(i T+\tau)-\sum_{i} q_{-m-1 / 2}^{2}(i T+\tau), \\
& R_{1, b}(\tau, m T)=\sum_{i} q_{0}(i T+\tau) \sum_{l} q_{0}(l T+m T+\tau) \\
& \quad+\sum_{i} q_{0}(i T+\tau-T / 2) \sum_{l} q_{0}(l T+m T+\tau-T / 2) \\
& \quad-\sum_{i} q_{0}(i T+\tau) \sum_{l} q_{0}(l T+m T+\tau-T / 2) \\
& \quad-\sum_{i} q_{0}(i T+\tau) \sum_{l} q_{0}(l T-m T+\tau-T / 2),
\end{aligned}
$$

$$
\begin{aligned}
& R_{1, c}(\tau, m T)=\left(\sum_{i} q_{m}(i T+\tau)\right)^{2}+\left(\sum_{i} q_{m}(i T+\tau-T / 2)\right)^{2} \\
& -\left(\sum_{i} q_{m-1 / 2}(i T+\tau)\right)^{2}-\left(\sum_{i} q_{-m-1 / 2}(i T+\tau)\right)^{2}
\end{aligned}
$$

$$
\begin{aligned}
& R_{2, a}(\tau, m T)=\sum_{i} q_{m-1 / 4}^{2}(i T+\tau)+\sum_{i} q_{m-1 / 4}^{2}(i T+\tau-T / 2) \\
& -\sum_{i} q_{m-3 / 4}^{2}(i T+\tau)-\sum_{i} q_{-m-1 / 4}^{2}(i T+\tau-T / 4) \\
& R_{2, b}(\tau, m T)=\sum_{i} q_{0}(i T+\tau) \sum_{l} q_{0}(l T+m T+\tau-T / 4)+ \\
& \quad+\sum_{i} q_{0}(i T+\tau-T / 2) \sum_{l} q_{0}(l T+m T+\tau-3 T / 4)+ \\
& \quad-\sum_{i} q_{0}(i T+\tau) \sum_{l} q_{0}(l T+m T+\tau-3 T / 4)+ \\
& \quad-\sum_{i} q_{0}(i T+\tau-T / 4) \sum_{l} q_{0}(l T-m T+\tau-T / 2)
\end{aligned}
$$

$$
\begin{aligned}
& R_{2, c}(\tau, m T)=\left(\sum_{i} q_{m-1 / 4}(i T+\tau)\right)^{2} \\
& +\left(\sum_{i} q_{m-1 / 4}(i T+\tau-T / 2)\right)^{2}-\left(\sum_{i} q_{m-3 / 4}(i T+\tau)\right)^{2} \\
& \quad-\left(\sum_{i} q_{-m-1 / 4}(i T+\tau-T / 4)\right)^{2}
\end{aligned}
$$

where

$$
q_{p}(a)=v(a) v(a+p T) .
$$




\section{REFERENCES}

[1] H. Sun and K.-T. Wu, "Clock recovery and jitter sources in coherent transmission systems," in Proc. OFC/NFOEC, Los Angeles, CA, 2012, paper OTh4C.1.

[2] D. Zibar, A. Bianciotto, Z. Wang, A. Napoli, and B. Spinnler, "Analysis and dimensioning of fully digital clock recovery for $112 \mathrm{~Gb} / \mathrm{s}$ coherent polmux QPSK systems," in Proc. ECOC, Vienna, Austria, 2009, paper 7.3.4.

[3] M. Kuschnerov, F. N. Hauske, K. Piyawanno, B. Spinnler, M. S. Alfiad, A. Napoli, and B. Lankl, "DSP for coherent single-carrier receivers," $J$. Lightw. Technol., vol. 27, no. 16, pp. 3614-3622, Aug. 2009.

[4] L. Barletta, R. Disarò, M. Magarini, and A. Spalvieri, "Post-filter optimization in timing recovery based on square-law detection," IEEE Photon. Tech. Lett., vol. 25, no. 9, pp. 821-824, May 1, 2013.

[5] L. Barletta, M. Magarini, F. Scardoni, and A. Spalvieri, "Impact of loop delay on the performance of Gardner timing recovery," IEEE Photon. Tech. Lett., vol. 25, no. 18, pp. 1797-1800, Sept. 15, 2013.

[6] M. Morsy-Osman, M. Chagnon, Q. Zhuge, X. Xian, and D. V. Plant, "Non-data-aided feedforward timing recovery for flexible transceivers employing PDM-MQAM modulations," in Proc. OFC, San Francisco, CA, 2014, paper W3B-4.

[7] C. R. Fludger, T. Duthel, P. Hermann, and T. Kupfer, "Jitter tolerant clock recovery for coherent optical receivers," in Proc. OFC/NFOEC, Anaheim, CA, 2012, paper OTh1F.3.

[8] M. Oerder and H. Meyr, "Digital filter and square timing recovery," IEEE Trans. Commun., vol. 36, no. 5, pp. 605-612, May 1988.

[9] W. P. Zhu, Y. Yan, M. O. Ahmad, and M. N. S. Swamy, "Feedforward symbol timing recovery technique using two samples per symbol," IEEE Trans. Circuits Syst. I, Reg. Papers, vol. 55, no. 11, pp. 2490-2500, Nov. 2005.

[10] S. J. Lee, "A new non-data-aided feedforward symbol timing estimator using two samples per symbol," IEEE Commun. Lett., vol. 6, no. 5, pp. 205-207, May 2002.

[11] Y. Wang, E. Serpedin, and P. Ciblat, "An alternative blind feedforward symbol-timing estimator using two samplers per symbol," IEEE Trans. Commun., vol. 51, no. 9, pp. 1571-1581, Sept. 2003.

[12] K. Shi, Y. Wang, and E. Serpedin, "On the design of a digital blind feedforward, nearly jitter-free timing-recovery scheme for linear modulations," IEEE Trans. Commun., vol. 52, no. 9, pp. 1464-1469, Sept. 2004.

[13] F. M. Gardner, "A BPSK/QPSK timing error detector for sampled receivers," IEEE Trans. Commun., vol. 34, no. 5, pp. 423-429, May 1986.

[14] A. Spalvieri and M. Magarini, "Wiener's analysis of the discrete-time phase-locked loop with loop delay," IEEE Trans. Circuits Syst. II, Exp. Briefs, vol. 55, no. 6, pp. 596-600, June 2008.

[15] W. Gappmair, "Self-noise performance of zero-crossing and Gardner synchronisers applied to one/two-dimensional modulation schemes," IEE Elec. Lett., vol. 40, no. 16, pp. 1010-1011, 5th Aug. 2004.

[16] A. Spalvieri and M. Magarini, "Minimum timing jitter in timing recovery based on pre-filtered square-law timing detection," in Proc. CTRQ, Colmar, France, 2009, pp. 46-51.

[17] A. N. D'Andrea and M. Luise, "Design and analysis of a jitter-free clock recovery scheme for QAM systems," IEEE Trans. Commun., vol. 41, no. 9, pp. 1296-1299, Sep. 1993.

[18] C.-Y. Lin, F. Grillot, Y. Li, R. Raghunathan, and L. F. Lester, "Characterization of timing jitter in a $5 \mathrm{GHz}$ quantum dot passively mode-locked laser," Opt. Express, vol. 18, no. 21, pp. 21932-21937, Sept. 2010.

[19] K. Yvind, D. Larsson, L. J. Christiansen, C. Angelo, L. K. Oxenløwe, J. Mørk, D. Birkedal, J. M. Hvam, and J. Hanberg, "Low-jitter and highpower 40-GHz all-active mode-locked lasers," IEEE Photon. Tech. Lett., vol. 16, no. 4, pp. 975-977, Apr. 2004.

[20] N. Ben-Hamida, J. Sitch, P. Flemke, D. Pollex, P. Schvan, Y. Greshishchev, S.-C. Wang, and C. Falt, "Clock recovery for a $40 \mathrm{~Gb} / \mathrm{s}$ QPSK optical receiver," in Proc. ICECS, Yasmine Hammamet, Tunisia, 2009, pp. 695-698.

[21] F. E. Scardoni, "Optimization of the post-detection filter in symbol timing-synchronization based on square-law detection for digital receivers," (in Italian), M.S. thesis , Scuola di Ingegneria Industriale e dell'Informazione, Politecnico di Milano, Milan, Italy, 2014. 